\title{
Fragmentation Functions and Implications for Spin Physics
}

\author{
Stefan Kretzer \\ Physics Department and RIKEN-BNL Research Center, Brookhaven National Laboratory, \\ Upton, New York 11973, U.S.A.
}

\begin{abstract}
The present status of fragmentation function (FF) analysis is summarized and the role of
\end{abstract} FFs in QCD hard scattering phenomenology is outlined with emphasis on spin physics.

\section{FF $\leftrightarrow$ PDF}

Parton distribution functions (PDFs) can be introduced through the local operator product expansion (OPE) in fully inclusive DIS - where PDFs emerge as the Mellin inverse of twist-2 operators. Fragmentation functions, on the other hand, relate to processes with one observed hadron in the final state (i.e. semi-inclusive) where the local OPE does not apply. FFs can, nevertheless, be defined in terms of the fields of the QCD Lagrangian within the generalized factorization theorems of QCD [1] or through cut vertices [2]. FFs are, therefore, part of a solid theory and no more model dependent than PDFs. Intuitively, the FF $D_{f}^{h}(z)$ represents a probability density that after a hard scattering event the parton $f$ turns into hadron $h$ with fractional momentum $z$ of the parton [3].

\section{OPERATIVE ROLE OF FFS FOR SPIN PHYSICS}

The fragmentation process turns perturbatively produced partons into non-perturbative hadronic bound states. The structural wealth encoded in this process is very rich - in part because independent functions are generated through the relative spin directions of partons and hadrons and through the presence of transverse momentum when the hadron is not collinear to the fragmenting parton. We refer the interested reader to the database [4] as a point of entry into the corresponding literature. In this contribution to the proceedings, without any further specification we will use the term fragmentation function more traditionally - as describing the production of unpolarized (or spin0 ) hadrons that are collinear with the fragmenting parton. These types of FFs are most important in their operative role of understanding hard scattering phenomena with identified hadrons in the final state at a quantitative level: Within factorized perturbative QCD we have that a hard scattering cross section $\sigma$ with hadrons in the initial and final state can be written as

$$
\sigma=\mathrm{PDF} \otimes \hat{\sigma} \otimes \mathrm{FF}
$$


with the symbol $\otimes$ denoting a convolution integral and $\hat{\sigma}$ being a partonic hard scattering cross section. In spin physics, the strategy is to fix the FFs in unpolarized reactions where the unpolarized PDFs are well known by now - and then later to "divide out" the FFs in polarized measurements to asses the spin dependent PDFs.

\section{RELEVANT PROCESSES: EXTRACTION AND APPLICATION}

Below I will list some of the relevant process to analyze and apply FFs. The page limitation does not allow the inclusion of many figures so I will make some quantitative claims without proof, referring the reader to the literature for details.

\section{$e^{+} e^{-}$Annihilations: $e^{+} e^{-} \rightarrow h X$}

The extraction of FFs [5] comes dominantly from the QCD analysis of $e^{+} e^{-}$annihilations where the QCD of the final state (FF) is not intertwined with initial state (PDF) effects. A lot of information can be obtained from e.g. the high precision LEP data. Limitations of $e^{+} e^{-}$are, however:

- The leading order partonic process $e^{+} e^{-} \rightarrow q \bar{q}$ produces quarks only. Not much can be learnt about gluon fragmentation

- Statistics drops low toward the leading particle tail of the spectrum limiting information on large- $z$ fragmentation.

- The internal light flavour structure cannot be disentangled. A systematic investigation [6] shows that mainly the flavour singlet (sum over flavours) FF is determined.

Because of these limitations FFs are not too well determined yet, even though high precision LEP $e^{+} e^{-}$data can be reproduced very successfully.

\section{Gluon Jets in $e^{+} e^{-}$}

Gluon jets can be identified by anti-tagging the " $g$ " in three jet $e^{+} e^{-} \rightarrow b \bar{b} g$ configurations. Within the parton model, this process simply measures the gluon fragmentation function. A NLO QCD global analysis of anti-tagged gluon jets is a formidable task, though, which has not been achieved so far.

\section{High $p_{\perp}$ Particle Production in Hadronic Collisions: $p p \rightarrow h X$}

In figures 1, 2 we perform a decomposition of $p p \rightarrow h\left(p_{\perp}\right) X$ for (central rapidity) collisions at RHIC [9] into the contributing initial state PDFs (fig. 1) and final state FFs (fig. 2). In fig. 3 we plot the $p_{\perp}$ dependence of the averaged scaling variables: Initial state partons carry fractional momentum $x_{1,2}$ and final state partons transfer fractional momentum $z$ in the hadronization process. Obviously, hadronic collisions 


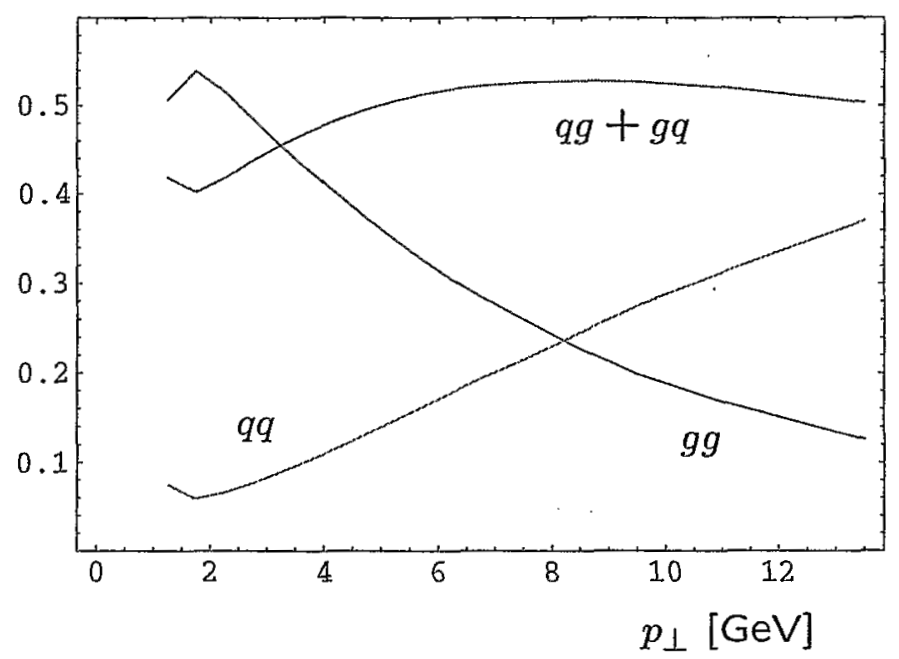

FIGURE 1. Initial state decomposition of (central rapidity) $p p \rightarrow \pi X$ at RHIC

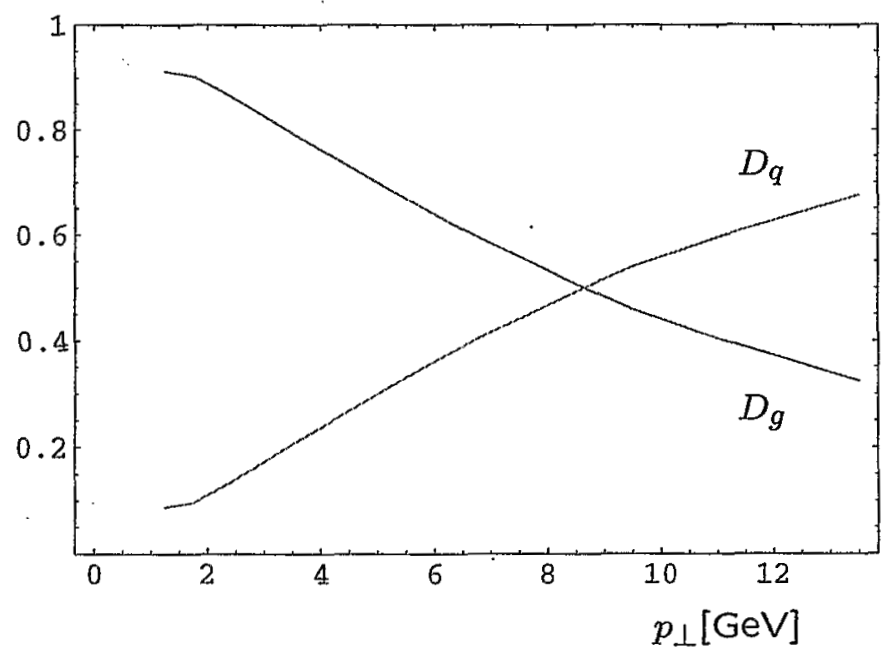

FIGURE 2. Final state decomposition of (central rapidity) $p p \rightarrow \pi X$ at RHIC

have the potential to provide information on large- $z$ fragmentation as well as on gluon fragmentation, two shortcomings of the $e^{t} e^{-}$process.

\section{Semi-Inclusive Deep Inelastic Scattering: $e N \rightarrow h X$}

SIDIS has the potential of disentangling the light flavour sector of the fragmentation process [6]. Following their "operative role" outlined above, one can then apply the FFs to polarized SDIS and disentangle the flavour structure of the longitudinal quark spin [7]. Uncertainties for the FFs (quantified in [6]) feed into uncertainties of the polarized PDFs. While SIDIS is a very promising process, a few obstacles of data at present 


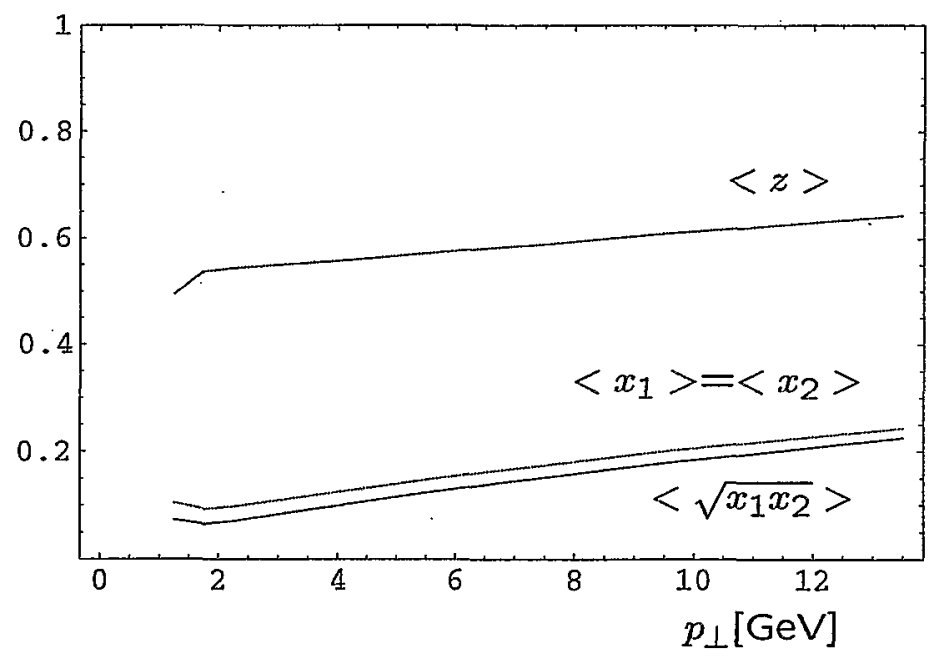

FIGURE 3. Average scaling variables

energies [8] will have to be better understood before it can be considered a bona fide lading twist perturbative reaction.

\section{ACKNOWLEDGMENTS}

It is a pleasure to thank E. Leader and E. Christova for collaboration and W. Vogelsang for discussions. I am grateful to RIKEN, Brookhaven National Laboratory and the U.S. Department of Energy (contract No. DE-AC02-98CH10886) for providing the facilities essential for the completion of this work.

\section{REFERENCES}

1. J. C. Collins, D. Soper and G. Sterman, in A. H. Mueller, ed., Perturbative Quantum Chromodynamics (World Scientific 1989); J..C. Collins and D.E. Soper, Nucl. Phys. B193, 381 (1981), B213, 545 (1983) (E); B194 445 (1982).

2. A.H. Mueller, Phys. Rev. D18, 3705 (1978).

3. R.D. Field and R.P. Feynman, Nucl. Phys. B136, 1 (1978).

4. http://www.pv.infn.it/ radici/FFdatabase/

5. S. Kretzer, Phys. Rev. D62 (2000) 054001; B. Kniehl, G. Kramer and B. Pötter, Nucl. Phys. B582 (2000) 514; L. Bourhis, M. Fontannaz, J.P. Guillet, M. Werlen, Eur. Phys. J. C19 (2001) 89; see these articles also for reference to the experimental data.

6. S. Kretzer, E. Leader, E. Christova, Eur. Phys. J. C22, 269 (2001).

7. See J. Wendtland's (for the HERMES collab.) contribution to these proceedings.

8. HERMES Collab. (A. Airapetian et al.), Eur. Phys. J. C21 599 (2001); M. Glück, E. Reya, hep-ph/0203063; G.A. Navarro, R. Sassot, Eur. Phys. J. C28, 321 (2003); A. Kotzinian, Phys. Lett. B552, 172 (2003).

9. PHENIX Collab. (S.S. Adler et al.), hep-ex/0304038. 